\title{
POINT CLOUD GENERATION OF A BUILDING FROM CLOSE RANGE THERMAL IMAGES
}

\author{
A. Dlesk ${ }^{1, *}, \mathrm{~K}$. Vach $^{2}$ \\ ${ }^{1}$ Department of Geomatics, CTU in Prague - adam.dlesk@gmail.com \\ ${ }^{2}$ EuroGV s.r.o. - vach@eurogv.cz
}

Commission V, WG V/7

KEY WORDS: Photogrammetry, Thermal images, Close range photogrammetry, BIM

\begin{abstract}
:
Thermal inspection of a building is a very important part of facility management. This paper presents the methodology how to capture thermal images on a case of a family house, how to process thermal images using current photogrammetric method and how to achieve point cloud where each point is enhanced by information about temperature. This enhanced point cloud could be data for BIM or GIS and could be a valuable result for facility managers where they can detect thermal leaks or anomalies. Due to the point cloud, the thermal leaks or anomalies could be easily located and described. The accuracy of the point cloud is tested by check points and the resulted point cloud is compared with point cloud gathered by a laser scanner. The last part of the paper is devoted to a discussion about the results and outputs of the photogrammetric processing of thermal images.
\end{abstract}

\section{INTRODUCTION}

Thermal inspection is a very important part of facility management of a building during its life cycle. Facility managers often need to thermally inspect the facilities to detect possible construction problems or to avoid significant heat leakage. This inspection is carried out by calibrated thermal cameras. The output of the thermal camera is a thermal image wherein each pixel is stored temperature value. If there is a need to localize the thermal phenomena (e.g. thermal leak) or if the facility managers need to get a general overview of thermal behaviour of the facility, the thermal images must be photogrammetrically processed. Due to the photogrammetric processing of thermal images, we can gather results such as a point cloud where each point of the point cloud is spatially located and contains information about temperature which is derived from original thermal images. Another result of photogrammetric processing of thermal images can be an orthophoto, where information about temperature can be stored in each pixel of the orthophoto. These results (point cloud and orthophoto) enriched by temperature information can be used for advanced spatial thermal analysis or for modelling heat propagation through investigated objects during the time. Thermal images are different from the RGB images and for photogrammetric processing, it is necessary to follow a different method. Thermal images have in general much lower resolution (usually between $160 \times 120$ pix to $640 \times 480$ pix) and some visible features of the objects are not detectable on thermal images. Because of that, the problem with signalizing classic control points commonly used in photogrammetry comes up. Also, software based on SfM (structure from motion) method could have a significant problem to detect tie points on thermal images. Published papers have shown the possibility to photogrammetric processing of thermal images usually captured using RPAS platform (Hoegner et al., 2016). Considering the usage of thermal imaging in facility management and in BIM, thermal images are often captured from the ground from a close distance. Terrestrial thermal images are also possible to photogrammetrically process (Scaioni et al., 2017). As was shown in the previous paper, to achieve 3D result (point cloud) where is also stored preserved temperature from thermal images, it is necessary to use a certain method (Dlesk et al., 2018). The results of photogrammetric processing of thermal images could be an important input to any BIM systems and help to manage the facilities.

\section{CAPTURING OF THERMAL IMAGES}

Thermal imaging is a sophisticated work and should be done by an experienced worker in a proper weather condition. A Lot of undesirable factors affect thermal imaging. Thermal inspection of a building or facades is usually carried out during significantly lower air temperature then temperature inside of an object and investigated object should not be under direct sunlight. The measurement should be always checked by touch thermometers.

\subsection{Thermal camera}

In this paper, for capturing thermal images was used thermal camera FLIR E95 with uncooled microbolometer sensor. This camera is also equipped with RGB sensor and with each thermal image camera captures the corresponding RGB with resolution 1280x960 pix and a wider field of view.

\begin{tabular}{|l|c|}
\hline Focal length & $10 \mathrm{~mm}$ \\
\hline Resolution & $464 \times 348$ pix \\
\hline Pixel pitch & $17 \mu \mathrm{m}$ \\
\hline Spectral range & $7.5-14.0 \mu \mathrm{m}$ \\
\hline FOV & $42^{\circ} \times 32^{\circ}$ \\
\hline Temperature accuracy & $\pm 2^{\circ} \mathrm{C}$ \\
\hline
\end{tabular}

Table 1. Parameters of thermal imaging camera FLIR E95.

\footnotetext{
* Corresponding author
} 


\subsection{Signalization of control points and check points}

In general, visible parts are not detectable on thermal images. Only temperature changes are visible on thermal images. Because of that problem with signalization of control points comes up. There are two ways how to signalize control points to be visible on thermal images.

1. Active control points

2. Passive control points

Active control points are warmed or cooled to get a different temperature of control point from the temperature of the surrounding part around the control point. Due to that, control points are visible on thermal images. The Second method is to use passive control points. Passive control points are made from material with different emissivity then the material on the object. Most of the building materials have very high emissivity (more than 0.9). In this case, it is possible to use aluminium foil as a material with low emissivity and suitable for control points. Aluminium foil was used for control points and check points for thermal imaging of the test object in this paper. Control points and check points were equally distributed on the test object. The points were geodetically measured by total station.

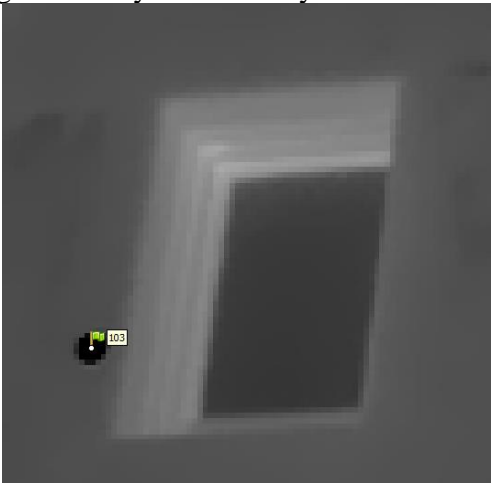

Figure 1. Passive control point visible on thermal image.

\subsection{Test object}

As a test object was chosen a two-floor family house which was easily accessible and was possible to capture thermal images all around. The family house was in a certain stage of reconstruction so there was an assumption that thermal images of the building will have a contrast and will be possible to process them using SfM method.

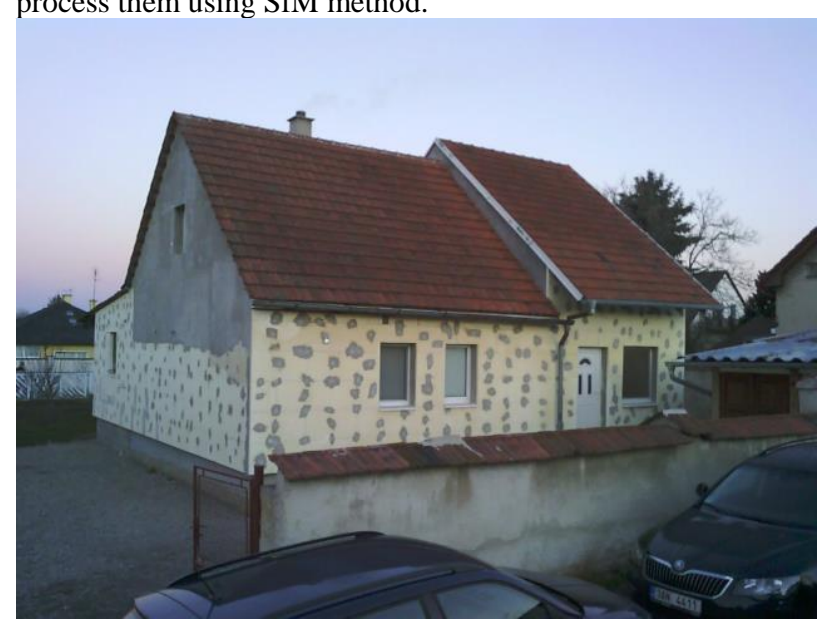

Figure 2. Test object.

\subsection{Capturing of thermal images}

Thermal images were captured all around the test object with very high overlap in two height levels (the first level was on the ground, the second level was $2 \mathrm{~m}$ above the ground). The sampling distance of the thermal images was chosen $1 \mathrm{~cm} / \mathrm{pix}$. In total, 581 thermal images of the object were captured.

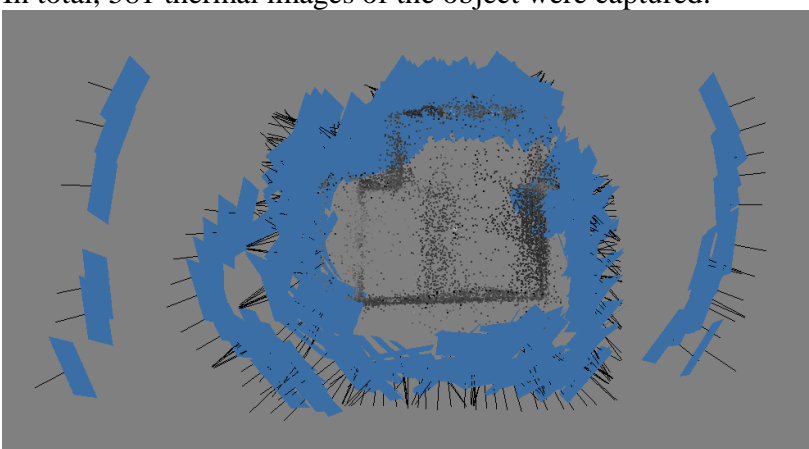

Figure 3. Visualisation of configuration of captured therma images.

\section{PHOTOGRAMMETRIC PROCESSING OF THERMAL IMAGES}

Thermal images were photogrammetrically processed using SfM method. In order to process thermal images using SfM method and to keep thermal information for 2D and 3D results, the following method was proposed.
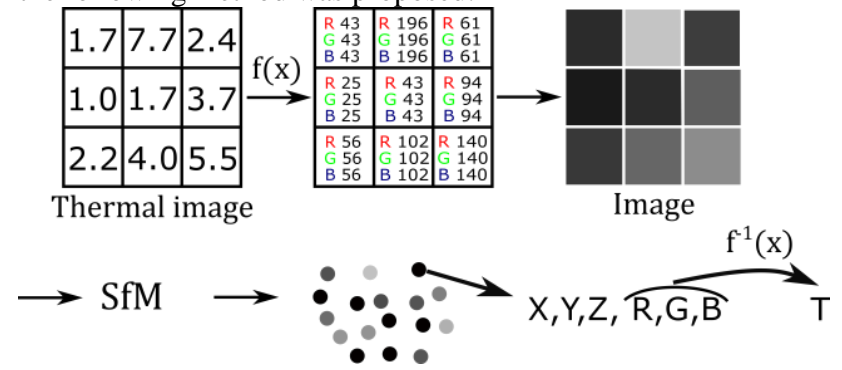

Figure 4. Workflow of photogrammetric processing of thermal images.

Thermal image, as an image with temperature value in each pixel should be converted to the more common image format (e.g. JPEG) to be processable in common software using SfM method. The conversion is made by stretching the histogram function of the thermal image to 8-bit. If the converted image has sufficient contrast, it is possible to process the images using $\mathrm{SfM}$ method and get relatively accurate results such as point cloud or orthophoto. Using the inverse function, it is possible to derive temperature value from colour of each point of the point cloud or each pixel of the orthophoto.

\subsection{Processing in Agisoft Photoscan}

For photogrammetric processing of thermal images using SfM method was chosen Agisoft Photoscan. 474 from 581 thermal images were coregistered. The reason why some of the images were not coregistered was that the images captured only a small part of the object where was almost no difference in the temperature and the image had almost no contrast. For georeferencing were used 9 control points and bundle adjustment was calculated. After the bundle adjustment, accuracy was checked on 11 checked points. The largest error of the check points was $0,037 \mathrm{~m}$. Total RMSE of the check points was $0,019 \mathrm{~m}$. 


\begin{tabular}{|c|c|c|c|c|}
\hline $\begin{array}{c}\text { Num. of } \\
\text { GCPs }\end{array}$ & $\begin{array}{c}\text { X Error } \\
{[\mathrm{m}]}\end{array}$ & $\begin{array}{c}\text { Y Error } \\
{[\mathrm{m}]}\end{array}$ & $\begin{array}{c}\text { Z Error } \\
{[\mathrm{m}]}\end{array}$ & $\begin{array}{c}\text { Total } \\
{[\mathrm{m}]}\end{array}$ \\
\hline 9 & 0,004 & 0,002 & 0,001 & 0,005 \\
\hline
\end{tabular}

Table 2. Control points - RMSE values.

\begin{tabular}{|c|c|c|c|c|}
\hline $\begin{array}{c}\text { Num. of } \\
\text { Check p. }\end{array}$ & $\begin{array}{c}\text { X Error } \\
{[\mathrm{m}]}\end{array}$ & $\begin{array}{c}\text { Y Error } \\
{[\mathrm{m}]}\end{array}$ & $\begin{array}{c}\text { Z Error } \\
{[\mathrm{m}]}\end{array}$ & $\begin{array}{c}\text { Total } \\
{[\mathrm{m}]}\end{array}$ \\
\hline 11 & 0,009 & 0,013 & 0,012 & 0,019 \\
\hline
\end{tabular}

Table 3. Check points - RMSE values.

\subsection{Results}

The result of thermal processing is a point cloud where each point of the point cloud is localized and enriched by temperature value. The enriched point cloud could be used as data to BIM, to facility management systems or for further spatial analysis in all kind of industries.

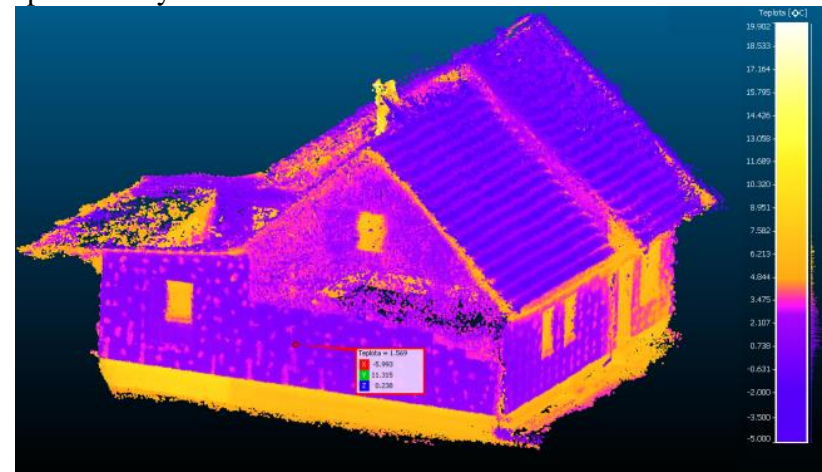

Figure 5. The point cloud where each point is enriched by temperature value.

Analogously, it is possible to use the suggested method to an orthophoto. Orthophoto where is stored thermal information in each pixel could be a good tool for facility managers how to thermal inspect building facade in a different stage of the lifecycle of a building. Due to the orthophoto is easy to detect thermal anomalies such us thermal leaks, localize thermal leaks and determine the range of thermal leaks.

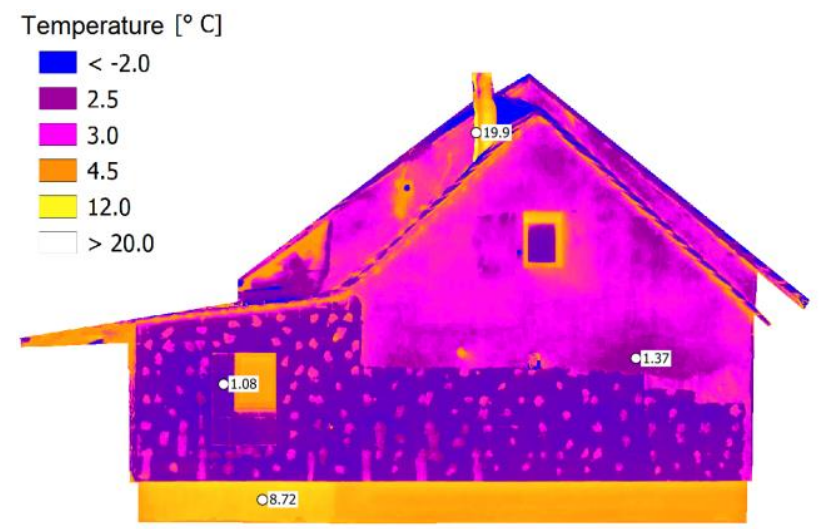

Figure 6. Orthophoto where temperature value is stored in each pixel.

\subsection{Comparison with laser scanning data}

The test object was surveyed by laser scanner Leica BLK360. The laser scanning data were used for comparison between point cloud generated using SfM method from thermal images and point cloud from laser scanner. The following figure shows cloud-to-cloud distances. It is possible to see some parts in the middle of the facades where the point cloud is very thin. Some parts of the point cloud created from thermal images are poor and some parts show a significant error.

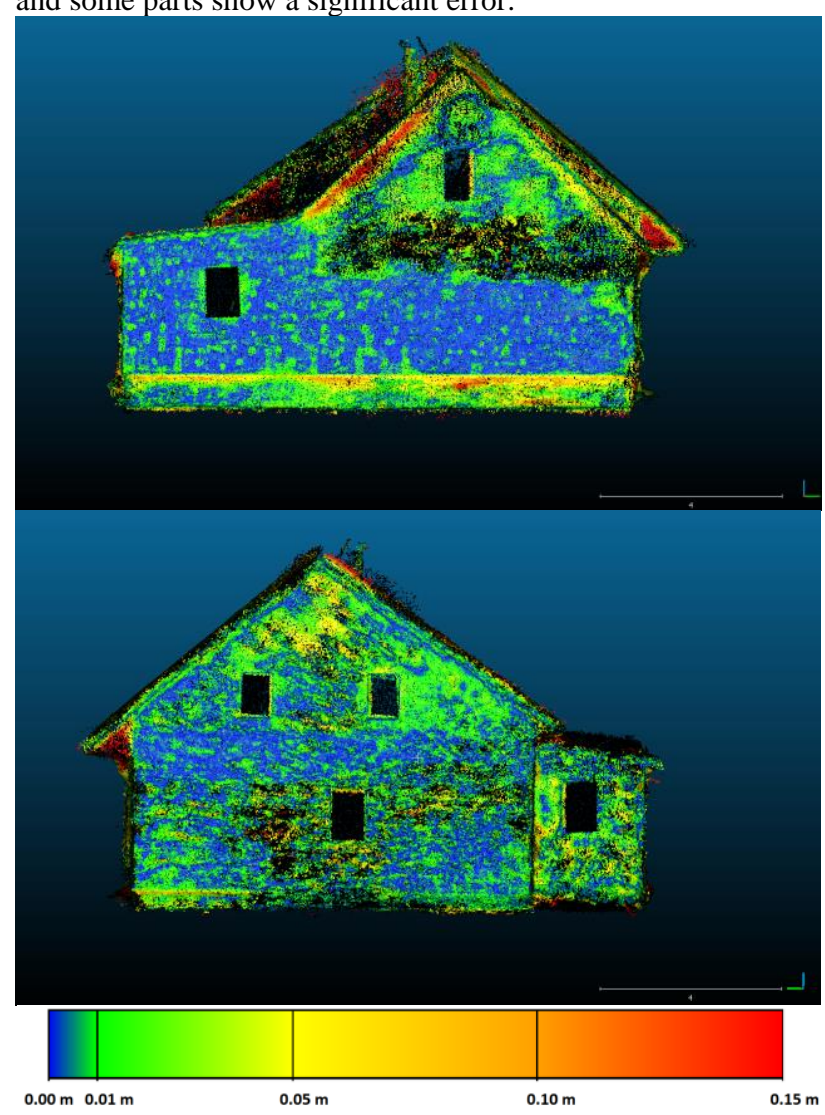

Figure 7. Comparison of the point cloud from thermal images and Leica BLK360 laser scan.

RGB images captured by thermal camera Flir E95 were processed using SfM method as well. The generated point cloud was also compared to the point cloud created by laser scanner Leica BLK360. The following figure shows a cloud-to-cloud comparison between point cloud generated from RGB images and point cloud from laser scanner. The comparison shows that point cloud generated from RGB images has higher quality than point cloud generated from thermal images. Point cloud generated from RGB images is lack of thin part and is denser in general and is more accurate in some parts then point cloud generated from thermal images. This experiment showed that fusion of thermal images and RGB images captured by the same camera could improve the results. In the next chapter possible methods how to process some kind of fusion of thermal images and RGB images are suggested. 

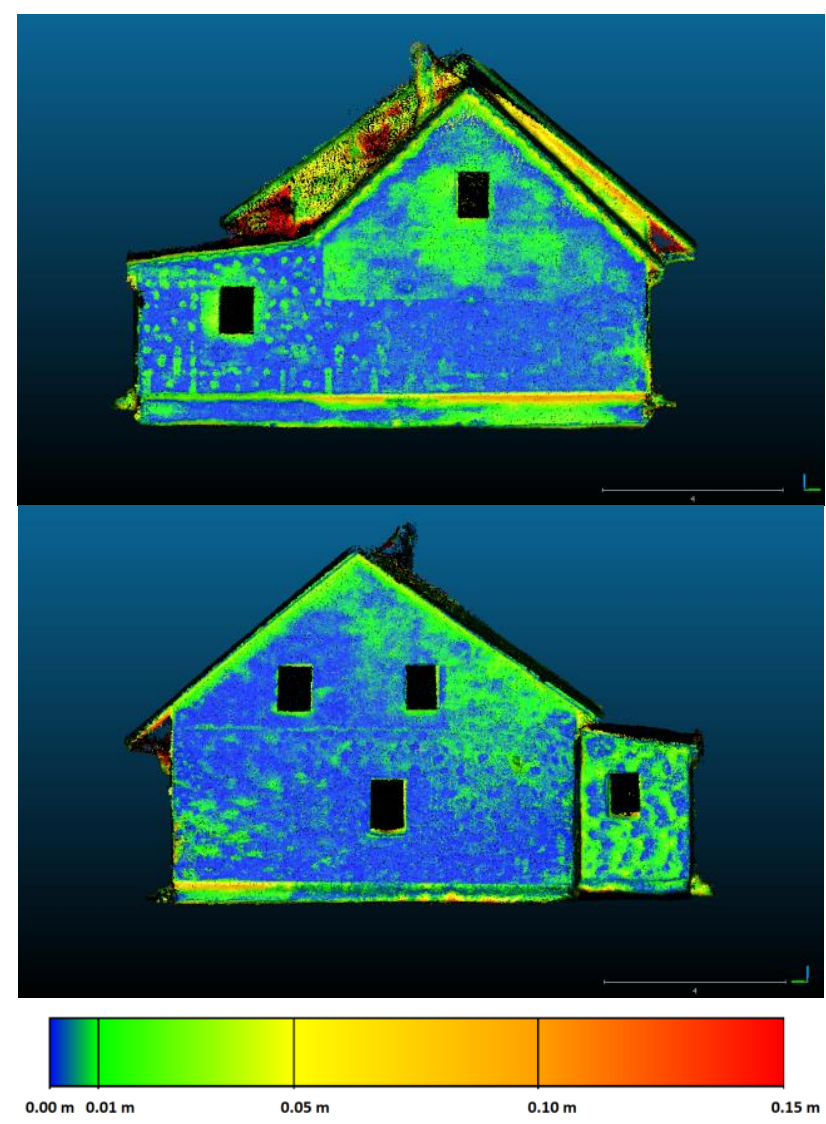

Figure 8. Comparison of point cloud from RGB images captured by thermal camera FLIR E95 and Leica BLK360 laser scan.

\section{FUTURE WORK}

If the captured thermal images have enough contrast and proper overlap, it is possible to process them photogrammetrically using SfM method. If the thermal images do not have enough contrast for processing with SfM method, it is probable to get only poor and inaccurate results. It is necessary to proposed methods which are based on a fusion of thermal images and RGB images. Some of the thermal cameras are also equipped with RGB sensor and to each thermal image camera captures also RGB image. These images could be used for the fusion. If the thermal camera is not equipped with an RGB sensor, it is possible to add a digital RGB camera to the thermal camera in a fixed configuration. The fusion of thermal images and RGB images can improve the result of photogrammetric processing, reduces the problem with lack of contrast on thermal images and eliminates the problem with signalization of control points and check points.

\subsection{Sharpening}

Sharpening method uses the corresponding RGB image to each thermal image. If the configuration of the corresponding images is fixed for all dataset, it is possible to compute the key of transformation between the two corresponding images. Thermal images will be converted to the 8-bit and those values will replace one of the RGB band of corresponding image (e.g. blue band will be replaced by converted thermal values). Those preprocessed images will be processed using SfM method. The result of the processing is point cloud with false colour. For each point, from the band which was replaced is then possible to compute using inverse function temperature values and add the information to the corresponding point.

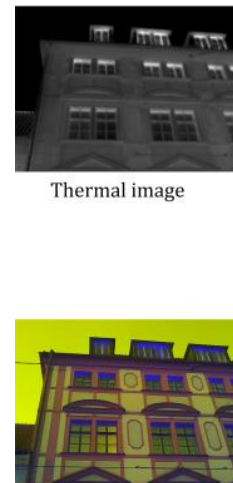

RGT

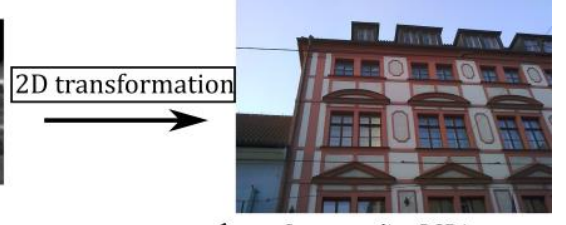

Corresponding RGB image

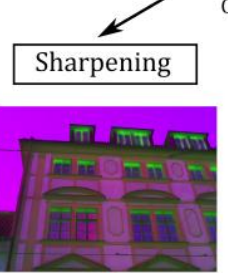

RTB

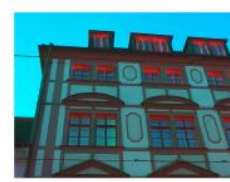

TGB

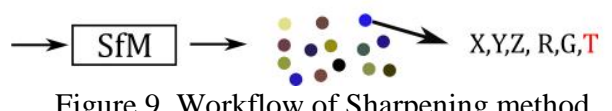

Figure 9. Workflow of Sharpening method.

\subsection{Reprojection}

Another way how to get point cloud with thermal information for each point is to process using SfM only RGB images. The result of the processing is coloured point cloud. Each point of the point cloud is possible to reproject to the RGB image. Then a test of visibility using depth map and normal map of RGB image must be done. If the reprojected point is visible on the RGB image it is possible to transform the point from the RGB image to the corresponding thermal image. From the pixel of the thermal image is subtracted temperature value and the temperature value is stored to the reprojected point in the point cloud. This method has an advantage over Sharpening method. In resulted point cloud from Sharpening method, there is one colour band discarded but in point cloud from Reprojection method all the colour bands are preserved.

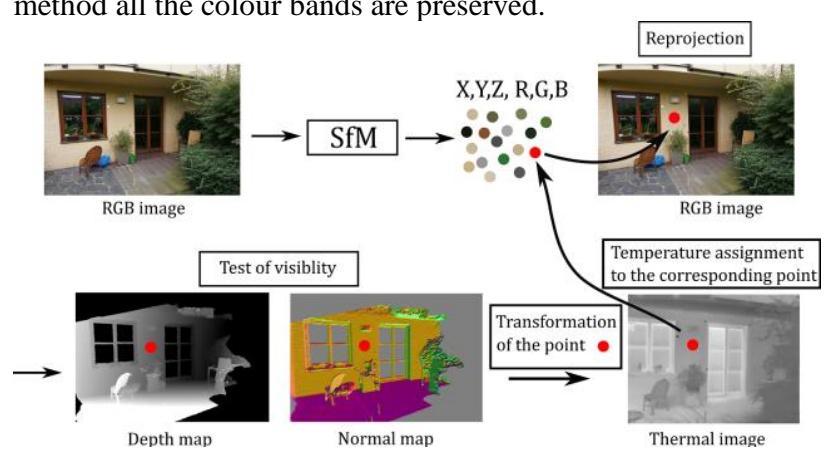

Figure 10. Workflow of Reprojection method.

\section{CONCLUSION}

During the life cycle of a building or a facility, it is needed to do thermal inspection of the building to detect thermal anomalies, such as thermal leaks. Detecting of thermal anomalies helps to locate construction problems and helps to reduce the costs of managing the building. If there is a need to locate the thermal anomalies and determine their range, thermal images must be photogrammetrically processed. Photogrammetric processing of thermal images gives result such as point cloud where each point is located in space and also can be enriched by temperature value derived from the original thermal image. The enriched point cloud is then valuable result 
for BIM systems which are used by facility managers. Thermal inspection is usually carried out by thermal cameras. Thermal images have in general much lower resolution than RGB images. Also, it is not possible to detect classic photogrammetric markers on the thermal image. Because of that, photogrammetric processing of thermal images brings challenges and it is necessary to use different methods of how to process thermal images. Experiments showed that it is possible to photogrammetrically process thermal images, generate point cloud with preserved information about temperature. If the thermal images are captured properly with sufficient overlap, with proper configuration and thermal images have contrast, it is possible to get relevant results. In this paper processing of thermal images is presented on the chosen test object. The coregistration of thermal images was checked on check points equally distributed around the test object. The largest error of the check points was $0,037 \mathrm{~m}$ and total RMSE was $0,019 \mathrm{~m}$. Then point cloud was generated and each point was enriched by temperature value derived from the original thermal image. This enriched point cloud was compared to the point cloud created by laser scanner Leica BLK360. The comparison shows a few imperfections. The point cloud generated from thermal images is very thin in some parts and in some parts (mainly upper parts and under the roof) the point cloud is relatively inaccurate.

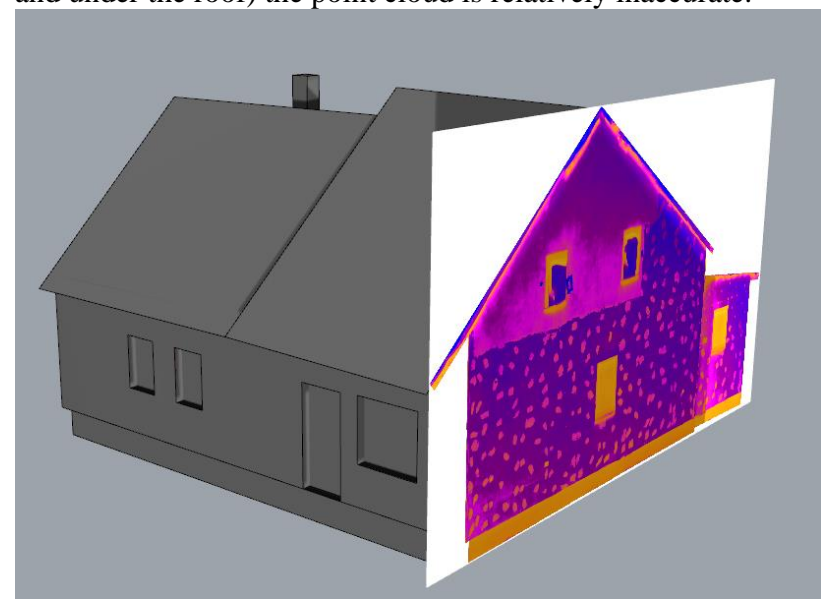

Figure 11. Orthophoto integrated to the 3D model of a test object. Orthophotos and Point clouds generated from thermal images can be valuable data for BIM systems.

If the result is poor and inaccurate it is possible to use a fusion between thermal images and RGB images. Modern thermal cameras have often RGB sensor in addition to the thermal sensor and for each thermal image camera captures also RGB image. This fact could be used for the fusion between the images and it is possible to process the fused images. This processing could give better results. In this paper, two methods of how to process thermal images and RGB images are presented. These methods will be tested in the following research.

\section{ACKNOWLEDGEMENTS}

This project was supported by grant of the Grant Agency of the Czech Technical University in Prague, grant No. SGS19/048/OHK1/1T/11. This project is a part of internal research of EuroGV s.r.o. company.

\section{REFERENCES}

Dlesk, A., Vach, K., Holubec, P., 2018: Usage of Photogrammetric Processing of Thermal Images for Civil Engineers. The International Archives of the Photogrammetry, Remote Sensing and Spatial Information Sciences, XLII-5, 99103. doi.org/10.5194/isprs-archives-XLII-5-99-2018.

Gade, R., Moeslund, T. B., 2014: Thermal cameras and applications: a survey. Machine vision and applications 25(1), 245-262.

Hoegner, L., Tuttas, S., Xu, Y., Eder, K., Stilla, U., 2016: Evaluation of methods for coregistration and fusion of RPASbased 3D point clouds and thermal infrared images. The International Archives of the Photogrammetry, Remote Sensing and Spatial Information Sciences, XLI-B3, 241-246. doi.org/10.5194/isprs-archives-XLI-B3-241-2016

Kadlec, K., 2014. Teoretické základy bezdotykového měření teploty (část 1). Automa, 2/2014, 13-15.

Kadlec, K., 2014. Teoretické základy bezdotykového měření teploty (část 2). Automa, 4/2014, 24-26.

Luhmann, T., Robson, S., Kyle, S., Harley, I., 2007: Close range photogrammetry. Wiley.

Scaioni, M., Rosina, E., L’Erario, A., Diaz-Vilariño, L., 2017: Integration of infrared thermography and photogrammetric surveying of built landscape. The International Archives of the Photogrammetry, Remote Sensing and Spatial Information Sciences, XLII-5/W1, 153-160. doi.org/10.5194/isprs-archivesXLII-5-W1-153-2017 